\title{
Artigo
}

\section{Triangulación y selectividad. ¿Por qué los países intermedios hacen cooperación sur-sur? Un estudio exploratorio desde el caso latinoamericano en Haití1 ${ }^{1}$}

Triangulação e seletividade. Por que os paises intermédios fazem cooperação sul-sul?

Um estudo exploratório desde o caso latino-americano no Haiti

Triangulation and selectivity. Why do intermediate countries deploy south-south cooperation? An exploratory study from the latin american case in Haiti

DOI: $10.5752 /$ P.1809-6182.2016v13.n2.p44

Bernabé Malacalza

\section{RESUMEN}

Recebido em: 10 março de 2016 Aprovado em: 07 de julho de 2016

El artículo analiza las motivaciones de la Cooperación Sur-Sur de los "paises intermedios" en los casos de Brasil, Venezuela, Argentina, México, Chile y Colombia en Haiti durante el periodo 2005-2015, considerando y comparando las correlaciones entre politica exterior, concepciones de desarrollo y Cooperación Sur-Sur. La hipótesis es que la CSS cambia a lo largo del tiempo en función de las distintas matrices de triangulación y selectividad que promueven los paises.

Palabras clave: Cooperación Sur-Sur. Paises Intermedios. Politica Exterior.

\section{RESUMO}

$O$ artigo analisa as motivaçôes da Cooperação Sul-Sul dos "paises intermédios" nos casos de Brasil, Argentina, México, Chile e Colômbia em Haiti durante o periodo 2005-2015, considerando e comparando as correlaçóes entre politica externa, concepçóes de desenvolvimento e Cooperação Sul-Sul. A hipótese é que a CSS muda ao longo do tempo em função das distintas matrizes de triangulação e seletividade que promovem os paises.

Palavras-chave: Cooperação Sul-Sul. Países Intermédios. Politica Externa

\section{ABSTRACT}

The article analyzes the motivations of South-South Cooperation provided by intermediate countries, taking into account the cases of Brazil, Venezuela, Argentina, Mexico, Chile and Colombia in Haiti for the period 2005-2015, considering and comparing the correlations between foreign policy, conceptions on development, and South-South Cooperation. The hypothesis is that SSC changes over time in relation to the matrixes of triangulation and selectivity that are promoted by different countries.

Key words: South-South Cooperation. Intermediate Countries. Foreign Policy.

1. Agradezco muy sinceramente la lectura, los comentarios y las críticas de los colegas evaluadores de la versión anterior de este texto, sin los cuales no hubiera podido advertir de la importancia de ahondar sobre los debates clásicos de las relaciones internacionales para abordar el objeto de este estudio de la CSS. Todos los comentarios confirman, además, una creencia previa: la necesidad de discutir y profundizar más los estudios aún incipientes sobre la CSS en América Latina.

2. Possui doutorado pela Latin American Faculty of Social Sciences (FLACSO), mestrado em Relaçóes Internacionais pela University of Barcelona, Spain, e pela University of San Andrés e FLACSO. Pesquisador assistente na National Scientific and Technical Research Council (CONICET) na Argentina, na National University of Quilmes (UNQ). Professor de cooperação internacional na National University of San Martin (UNSAM). orcid.org/00000003-4369-0534 


\begin{abstract}
"De una determinada causa dada se sigue necesariamente un efecto, $y$, por el contrario, si no se da causa alguna determinada, es imposible que un efecto se siga"

"El conocimiento del efecto depende del conocimiento de la causa, y lo implica"

"La potencia de un efecto se define por la potencia de su causa, en la medida en que su esencia se explica o define por la esencia de su causa"
\end{abstract}

(SPINOZA,1987).

\section{Introducción}

¿Qué son los países intermedios (PIs) y por qué hacen Cooperación Sur-Sur (CSS)? Los PIs son aquellos países que, no siendo decisivos en la configuración sistémica como las "grandes potencias", de una manera u otra intentan salir de la insignificancia. Para ellos, la CSS representa, pues, esa "aspiración de ascenso" que se traduce en el relacionamiento con los "grandes" y los "semejantes". Sin embargo, no existe una única motivación ni una única forma de articular la CSS, por lo que para comprenderla es necesario analizar y explorar sus variados y diversos "sentidos".

El objetivo de este artículo es analizar las motivaciones de los PIs latinoamericanos (Brasil, Venezuela, Argentina, México, Chile y Colombia) en el ámbito de la CSS, desde la ponderación de las diferentes dinámicas existentes entre esquemas de política exterior, concepciones del desarrollo y CSS. La contrastación de este supuesto se realiza sobre un estudio exploratorio del caso de la CSS latinoamericana en Haití durante los ańos 2005 y 2015, en un espacio de elevada actividad y protagonismo de los PIs en convivencia con una multiplicidad de donantes. Para ello, se parte de dos hipótesis de trabajo. La primera hipótesis está formulada en términos de intereses, la segunda en términos de ideas:

- la CSS actuó como un instrumento al servicio del interés de las políticas exteriores de los PIs latinoamericanos, procurando insertar distintos esquemas de triangulación proclives a cooperar con ciertos actores en desmedro de otros, en función de los distintos intereses y alcances de la proyección internacional de cada PI;

- la CSS sirvió como una plataforma de principios y prácticas para seleccionar y transferir en el escenario internacional concepciones de desarrollo formuladas a nivel doméstico, afianzadas en diferentes esquemas de relación público-privado y de intervencionismo estatal y en trayectorias de políticas publicas de acuerdo a las especializaciones de cada PI.

El contenido del artículo se subdivide en cuatro secciones: la primera sección recoge brevemente el debate en las relaciones internacionales sobre las motivaciones de la ayuda exterior; la segunda recala en el concepto de PIs y define las motivaciones de la CSS y las variables de análisis; la tercera analiza la relación entre política exterior y CSS en Haití de acuerdo a las variaciones en la "triangulación" (Hipótesis 1), la tercera se enfoca en el vínculo entre concepciones de desarrollo y CSS en Haití de acuerdo al análisis de la "selectividad" (Hipótesis 2); finalmente, se elaboran una serie de conclusiones.

\section{Ayuda exterior y relaciones internacionales}

La ayuda exterior ha sido objeto de interés de la teoría de las relaciones internacionales desde la década del sesenta. La vinculación de ésta con la política exterior se encuentra en la propia génesis del régimen de ayuda en sus primeras cuatro décadas tras la Segunda Guerra Mundial. Como sostiene Sogge (2002, p. 64), "ganar la Guerra Fría fue claramente el propósito", pero no ha sido ésta la única motivación de la ayuda. La discusión por identificar qué dosis de "interés" y cuáles de "al- 
truismo" contiene el suministro de ayuda externa ha sido - y continúa siendo - un motivo de división académica.

La vieja disputa entre realismo e idealismo en las relaciones internacionales sobre el comportamiento externo de los estados ha sido también reeditada en los estudios sobre la ayuda externa. La grandes preguntas de este debate tienen que ver con el porqué. ¿Cuáles son las motivaciones o el sentido de los suministros de ayuda externa? ¿Por qué se destinan paquetes de ayuda a unos países y no a otros? ¿Cuáles son los drivers y los estímulos que hacen posible la cooperación entre países?

Para Morgenthau (1962), el padre del realismo, la ayuda está dominada por el interés político del donante y es por ello que existen seis tipos de ayuda: la humanitaria, la de subsistencia, la militar, los sobornos, la de prestigio y la de desarrollo económico. Ésta última es ineficaz, porque sus resultados son inciertos e incluso pueden ser contraproducentes; en tanto que la de prestigio es preferible por sus efectos políticos inmediatos y visibles (es el caso de los grandes proyectos de infraestructura). En oposición al realismo, los liberales institucionalistas y los constructivistas sostienen argumentos parecidos entre sí. Keohane (2005) explica la cooperación como una respuesta a la necesidad de coordinación de políticas creada por la interdependencia, que, a la vez, es producto de la discordancia. Como la armonía no es posible y existen reales o potenciales fuentes de conflicto, los estados deciden cooperar y hacer "ajustes mutuos" para satisfacer demandas de otros. Aquí el eje está en la interacción, más que en el interés, pero sin desconocer la tensión de intereses divergentes.

Los constructivistas, por su parte, le asignan a la moral, la solidaridad y el altruismo un papel importante. Lumsdaine (1993) trata de demostrar que las orientaciones hacia la reducción de la pobreza en países con sistemas de bienestar social, las interacciones con otros estados y ciudadanos en la sociedad internacional y el principio de asistencia a aquellos con mayores necesidades han moldeado las "prácticas de ayuda". Para los constructivistas, las ideas más que los intereses han sido el núcleo. No obstante, podría resultar ingenuo si se pensara que sólo buenas ideas "altruistas" han moldeado al sistema. En ese sentido, Sogge (2002, p. 175) se pregunta: ¿y qué ha ocurrido con las malas ideas o las ideas peligrosas? El comercio y las alianzas políticas han guiado a la ayuda exterior, así como la ideología, formulando problemas y soluciones, etiquetando identidades y asignando valores. ${ }^{3}$

Si estuviéramos ante la necesidad de resumir este debate en dos miradas o perspectivas, no estaríamos lejos de entrever que se tratan de posiciones opuestas que viran entre lo cínico y lo ingenuo. Lo cierto es que las políticas exteriores son contenedoras tanto de intereses como de ideas y, por esa razón, no están exentas de contradicciones. Como respuesta a ese dilema, algunos autores sostienen que es mucho más útil incorporar en el análisis elementos de ambas perspectivas. Por ejemplo, entre los muchos "usos de la ayuda externa", Veen (2011) destaca siete: seguridad, influencia, riqueza, autointerés iluminado, reputación, obligación y humanitarismo. Estos cuadros de referencias son competitivos y su distinto peso relativo se refleja en las políticas que siguen los estados, lo que puede llevar a resultados ambiguos. Más allá de cualquier clasificación, como sostiene Sogge (2002, p. 64), "el régimen de ayuda no es coherente" y encierra "varias y diversas motivaciones que cambian a lo largo del tiempo y en función de los donantes".

¿Pueden estas grandes preguntas de las relaciones internacionales sobre la ayuda externa y sus

3. Según Sogge (2002, p. 67), esto es una realidad incluso en Suecia, "donde durante tiempo se supuso que la ayuda estaba guiada por inquietudes humanitarias. Los datos sugieren que Suecia actúa más bien como un comerciante que como un activista, incluso en los ochenta, cuando realizó importantes contribuciones al cambio político en el sur de África. 
motivaciones servir también para el análisis de la cooperación de los PIs? Aún cuando varíen las respuestas en función de los diferentes roles o comportamientos esperados de estos países en la escena internacional, las preguntas sobre el rol de los intereses y las ideas en la cooperación continúan siendo útiles. Sería absurdo negar que la CSS destinada a fomentar el desarrollo económico ha seguido consideraciones competitivas de interés y de principios similares a la ayuda externa.

\section{Las motivaciones de los países intermedios para hacer cooperación sur-sur}

¿Qué es un PI? ¿Qué se espera de un PI? ¿Cuál es el papel de los PIs en la cooperación al desarrollo? ¿Qué motivaciones estimulan la incursión de estos países en este campo? ¿Cómo y en qué difieren los PIs que hacen CSS? Así como han proliferado varias y diversas etiquetas para nominar a los países que se encuentran en la mitad de la pirámide de poder internacional ${ }^{4}$, tampoco existe un único tipo de PI ni de CSS. Habida cuenta de un vacío en la literatura, la clasificación y ponderación de estos en función de su papel como donantes constituye nuestra modesta contribución. ${ }^{5}$

4. El concepto de PIS PIs es esencialmente controvertido, esquivo y falto de claridad. Esta imprecisión se constata, por ejemplo, en la variedad de nomenclaturas utilizadas, ya sean "potencias medias o medianas" (HOLBRAAD, 1971), "potencias medias emergentes" (SCHOEMAN, 2000), "potencias emergentes" (JONES, HILDRETH, 1986), "estados pivote" (CHASE, HILL, KENNEDY, 1996), "potencias regionales" (NOLTE, 2010), "potencias regionales emergentes" (NEL, 2010), "eventuales grandes potencias" (HURRELL, 2006), entre otras.

5. Muy recientemente, casi al momento de cierre de este artículo, Santander Campos (2016) realizó un aporte novedoso y útil en ese sentido, al comparar la CSS de Chile, Venezuela y Brasil como referentes de tres modelos en los que convergen diferentes intereses e ideas sobre la CSS. Ello abona a la idea de convergencia en la agenda de investigación de la CSS, que como la propia dinámica política, se encuentra en proceso de debate y acción.
En su tesis doctoral, Huelsz (2009) identifica tres momentos en la conceptualizacion de los PIs. Durante la Guerra Fría, el concepto aparece ligado a una concepción normativa de la política exterior de potencias medianas como Canadá y Australia (CLAXTON, 1944; GLAZEBROOK, 1947; HOLBRAAD, 1984). Ya durante la Posguerra Fría, se lo asocia al estudio de dinámicas regionales, poniendo el foco en "países pivote" como México, Brasil, Egipto, Sudáfrica, Turquía, India, Pakistán e Indonesia (CHASE, HILL, KENNEDY, 1996). Finalmente, en el siglo XXI, reaparece un uso más frecuente de la nomenclatura "emergentes", siendo vinculada principalmente al ascenso geopolítico de los BRICS, con especial mención a sus proyecciones en Asia y África (HURRELL, 2006; BISCHOFF, 2003; RYERSON; DEWITT, 2006; NOLTE, 2007; FLEMES, 2010).

Si bien los estudios sobre los BRICS como actores individuales y colectivos se han vuelto de enorme valor, sólo recogen una mirada parcial, pues si bien los BRICS son emergentes, no todos hacen CSS (MUHR, 2016). Por caso, Rusia, un país de renta alta con aspiraciones de acceder al Comité de Asistencia al Desarrollo de la Organização para a Cooperação e Desenvolvimento Económico (OCDE), registra sus acciones como ayuda oficial al desarrollo; en tanto China está más bien cerca de considerarse una gran potencia que un PI.

De hecho, los BRICS constituyen un rara avis dentro de la categoría amplia de PIs. Existen otros países que, no siendo grandes potencias, también poseen cierta disposición a ejercer una influencia selectiva en determinados temas de la agenda internacional por medio de la CSS. Entre las razones para ejercer ese rol están las aspiraciones de ascenso en la pirámide de poder, pero también la puesta a prueba de la experiencia acumulada o la trayectoria en determinados temas de la agenda, como agroalimentos, medio ambiente, energías alternativas, etc. 
La literatura no acuerda sobre una única definción de PIs. De acuerdo con Lima (2007), el concepto de PIs ha sido utilizado con por lo menos tres significados diferentes: el objetivo, el subjetivo y el social. A su vez, estas clasificaciones poseen una fuerte analogía con las definiciones clásicas de poder: poder como posesión de recursos materiales, poder relacional o influencia, y poder sobre resultados. $\mathrm{Si}$ conjugamos los tres atributos identificados por Lima (2007) (objetivo, subjetivo y social) en una sola definición, arribamos al siguiente concepto:

a) Un PI es aquel que dispone de:

- una situación relativa de capacidades materiales que le permite ejercer cierta "generosidad" fuera de sus fronteras;

- un deseo o aspiración de un estatus de país emergente, traducido en el ejercicio de un liderazgo selectivo en determinados temas o "nichos" de la agenda internacional; y un poder de persuasión o "influencia legítima” en relación a un número razonable de estados, tanto de los más poderosos, como de los semejantes, que le permite obtener un reconocimiento de ese estatus.

¿Por qué los PIs hacen CSS? No existe pues una única motivación, aunque una respuesta común podría ser que, para ejercer influencia en la agenda internacional, los PIs suelen apoyarse más sobre bases de poder intangible o blando, como las ideas, la cultura y la comunicación. En ese sendero, un instrumento cada vez más frecuente es la diplomacia pública, un paquete de acciones destinado a "ganar mentes" y captar la atención del gran público de otros países sobre la política exterior (MELISSEN, 2005) y, dentro de esa caja, la CSS al desarrollo puede actuar como incentivo para proyectar

6. Por "influencia legítima” se entiende un concepto equivalente al de autoridad, una influencia aceptada o consentida por quienes la reciben en función de la reputación con la que cuenta el actor que la ejerce en determinada cuestión o tema (DAHL, 1976:64). la imagen del país en el exterior. La CSS, entonces, puede asumir la forma de subvenciones, créditos concesionales y no concesionales, asistencia técnica, apoyo presupuestario o alivio de deuda; mientras que los proyectos pueden oscilar entre la ambiciosa y costosa "ayuda de prestigio" (MORGENTHAU, 1962), que se materializa, por ejemplo, en grandes obras de infraestructura (estadios, vías férreas, autopistas, telecomunicaciones, etc.), y los resultados más lentos y menos visibles que ofrece la cooperación técnica horizontal.

Más allá de las semejanzas, existen diferencias en la CSS en términos de intereses y proyección. Los PIs pueden adscribir a diferentes visiones de mundo, elaborando diversos diagnósticos del sistema internacional y estableciendo, desde allí, distintos esquemas de inserción internacional y de CSS. Así entonces, están los PIs que aspiran a convertirse en grandes potencias; los que buscan reputación, prestigio, credibilidad internacional para "jugar en las grandes ligas" del Norte (globalistas adaptativos), los que pretenden ser una potencia del Sur o una voz del mundo en desarrollo (autonómicos reformistas) y los que plantean un deseo de cambio radical o anti-sistémico (autonómicos secesionistas o contestarios).

En términos de identidades e ideas, la CSS constituye también una oportunidad para transferir al ámbito internacional principios y prácticas que los PIs adoptan en el ámbito doméstico. En el caso de los PIs de mayor envergadura, como Brasil e India, la CSS se nutre del mayor potencial que disponen estos países para convertirse en grandes potencias, vinculándose con políticas exteriores de escala y de alto perfil internacional centradas en la búsqueda de acceso a mercados y la expansión de sus empresas transnacionales con el apoyo de sus bancos nacionales de desarrollo a través de formulas integradas que incluyen a las inversiones, la cooperación técnica y el comercio. En el resto de los PIs, con capacidades relativas menores, lo que 
abunda es la cooperación técnica de bajo coste, pero con una fuerte impronta de principios basada en una retórica de la horizontalidad, el consenso y la equidad. Aquí, la CSS se restringe al intercambio de conocimientos.

En este artículo, nos proponemos diferenciar a los PIs en función de sus políticas exteriores y, más precisamente, de sus políticas exteriores de CSS. Para ello estimamos como centrales dos variables de distribución de las acciones de cooperación: la triangulación y la selectividad. La primera se relaciona con la disposición de los PIs a triangular en sus políticas de CSS con otros actores, tales como organismos multilaterales, regionales, países del Norte y del Sur, organizaciones de la sociedad civil (OSC) y actores privados. La segunda con la tendencia de los PIs a especializarse en determinados temas o asuntos de las políticas públicas en función de sus trayectorias o experiencias acumuladas.

La triangulación en CSS depende fuertemente de los esquemas de política exterior que se asuman. Para el caso de los PIs latinoamericanos, ello se refleja en las diferentes posiciones de los países frente a la agenda de cooperación del Norte y ante el sistema de ayuda internacional en su conjunto. Hirst (2010) observa que podrían identificarse al menos tres tipos de respuestas: el pragmatismo autonómico, que busca reforzar las características peculiares de la CSS, acudiendo a una geometría variable, pero preservando márgenes de maniobra propios; el idealismo defensivo, que se esfuerza por dar un lugar preferencial a las triangulaciones Sur-Sur-Sur para contrarrestar la agenda del Norte; y la tercerización prestigiosa, que considera a la triangulación Norte-Sur-Sur como un marco de oportunidades para ampliar los vínculos con el mundo industrializado. Asimismo, estas opciones no son excluyentes y su superposición puede dar margen a políticas ambiguas.

La selectividad depende fuertemente de los modelos, visiones y enfoques sobre el desarrollo y las po- líticas publicas que se adopten. Además, los PIs pueden diferir en las concepciones y formas de aspirar a conservar, o bien de adquirir, cierta musculatura en las negociaciones multilaterales que les afectan. En ese sentido, la apuesta a la CSS, tanto en la construcción de coaliciones como en la cooperación técnica horizontal, está estrechamente relacionada con las capacidades especiales de estos países de influir en ciertos sectores de la agenda internacional, liderando ciertos espacios o introduciendo nuevos temas o enfoques en diversas áreas de intervención. Ello se correspondería con una estrategia similar a la de una "diplomacia de nicho" (niche diplomacy), a partir de la cual la política exterior invierte selectivamente en sectores/temas/agendas en los que cada país posee ventajas comparativas o experiencia acumulada (COOPER, 1997; POTTER, 1996)

Tanto la triangulación como la selectividad son variables que permiten recolectar datos sobre las proyecciones que, en términos de intereses e ideas, realizan los PIs en el escenario internacional a través de la CSS. ¿Qué significa la CSS en términos de intereses e ideas? ¿Cómo interactúa con las políticas exteriores de los PIs en un espacio particular? De aquí en adelante, se intentará responder a estas preguntas de forma exploratoria (no absoluta ni definitiva), tomando como caso de estudio a las políticas exteriores de CSS desplegadas por Brasil, Venezuela, Argentina, México, Chile y Colombia en Haití durante el período 2005-2015.

\section{El vínculo entre política exterior y cooperación sur-sur en Haití: la triangulación}

La presencia latinoamericana en Haití desde 2004, tanto por el envío de militares en el marco de la Misión de Estabilización de Naciones Unidas (MINUSTAH), como por la contribución de la región en materia de CSS, no tiene antecedentes de similar 
relevancia. Un total de doce países de la región enviaron contingentes a la misión ${ }^{7}$, llegando a representar el $61 \%$ de las tropas en 2010. Simultáneamente, siete países contribuyentes a la misión (Brasil, Argentina, México, Chile, Colombia, Ecuador y Perú) y dos no contribuyentes (Venezuela y Cuba) iniciaron proyectos de CSS (MALACALZA, 2016).

La relevancia metodológica de un estudio múltiple de caso sobre Haití se encuentra en que ofrece la posibilidad de explorar cuáles han sido los factores motivantes de la CSS en un espacio en el que convivieron una cantidad importante de grandes donantes y PIs. ${ }^{8}$ Se trata de un trabajo exploratorio orientado a definir preguntas e hipótesis para estudios posteriores más amplios (YIN, 1993). Los PIs seleccionados en esta muestra comparativa son Brasil, Venezuela, México, Argentina, Colombia y Chile. ${ }^{9}$ Asimismo, la muestra comparativa analizada se deriva de un catastro de proyectos elaborado por el autor para el Programa de Fortalecimiento de la CSS de la SEGIB (MALACALZA, 2016). Dicha base de datos contiene información relativa a los 360 proyectos y acciones de CSS desplegados por los países latinoa-

\begin{abstract}
7. Los países latinoamericanos contribuyentes de tropas militares en el período 2004-2015 fueron Brasil, Uruguay, Argentina, Chile, Perú, Bolivia, Guatemala, Ecuador, Paraguay, El Salvador, Honduras y México. Colombia realizó una contribución en efectivos policiales; México envió dos efectivos militares en 2015.

8. De acuerdo a datos publicados por órganos ejecutores de CSS de la región, se estima que durante la década 2005-2015, un total de 360 proyectos fueron impulsados por Venezuela (164 proyectos), Brasil (107), Argentina (30), México (23), Ecuador (13), Chile (13), Cuba (10), Colombia (5) y Perú (1) en territorio haitiano. En el período 2005-2010, los países latinoamericanos iniciaron 112 proyectos, un promedio de $22 \mathrm{al}$ año, en una tendencia en franco aumento. Después del terremoto, se produjo un salto exponencial con 165 nuevos proyectos en un solo ańo, 88 de ellos abocados a la recuperación de infraestructura (MALACALZA, 2016).
\end{abstract}

9. Además de los países mencionados, Cuba, Ecuador y Perú también desplegaron proyectos de cooperación en Haití. El caso de Cuba es emblemático, en razón de una trayectoria de más de veinte años en el campo de la cooperación en salud con Haití; sin embargo, por tratarse de un estado pequeño, no ha sido abordado en este estudio. Entretanto, los caso de Ecuador y Perú son de un calibre menor y más reciente. Uruguay, en tanto, no participó de acciones de CSS, aunque tuvo una destacada participación en el aporte de militares. mericanos en Haití entre 2005 y 2015, incluyendo a los proyectos de infraestructura.

Como puede apreciarse en el gráfico 1, las interacciones entre políticas exteriores y CSS de los PIs en Haití que se verifica en la variable "triangulación” da cuenta de la existencia de tres esquemas diferenciables en línea con lo planteado por Hirst (2010): el pragmatismo autonómico, el idealismo defensivo, y la tercerización prestigiosa.

El pragmatismo autonómico tendió a diversificar sus acciones de CSS en función de sus intereses de política exterior. En el caso de Brasil, la CSS adoptó un perfil más orientado al multilateralismo global, que se reflejó en una mayor inclinación a interactuar con organismos multilaterales del sistema de Naciones Unidas (41\% del total de las acciones). Ello se basó en una "política de vinculación de cuestiones" (linkage politics) coherente con su pertenencia al bloque BRICS y su aspiración a utilizar el liderazgo en la MINUSTAH como un modo de aproximación a su objetivo de acceder a un asiento permanente en el Consejo de Seguridad (MALACALZA, 2016). En el caso de Argentina, los objetivos no estuvieron tan claros, por lo que el margen de ambigüedad fue mayor. La alta diversificación de su CSS se explica por la gran atracción de su proyecto ProHuerta, que contó con un amplio y variado apoyo de organismos multilaterales y donantes tradicionales. Asimismo, el $46 \%$ de sus acciones fueron articuladas vía un organismo regional ad hoc, la Secretaría Técnica de UNASUR, en la que Argentina fue único contribuyente ${ }^{10}$. También registró proyectos con socios del Sur, entre los que

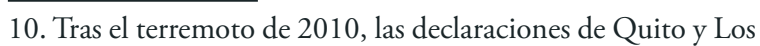
Cardales lograron un compromiso de 300 millones de dólares de los estados miembros de UNASUR para el funcionamiento de una Secretaría Técnica en Puerto Príncipe. Sin embargo, únicamente Argentina contribuyó a su funcionamiento, siendo responsable del presupuesto de casi 17 millones de dólares, a cargo del Embajador Especial de Argentina ante UNASUR y Representante Especial de esa organización en Haití, cuyo mandato respondió directamente al área política de la Cancillería de ese país. 


\section{Grófico 1- Porcentaje de acciones de CSS en Hoití} según actores socios (2005-2015)

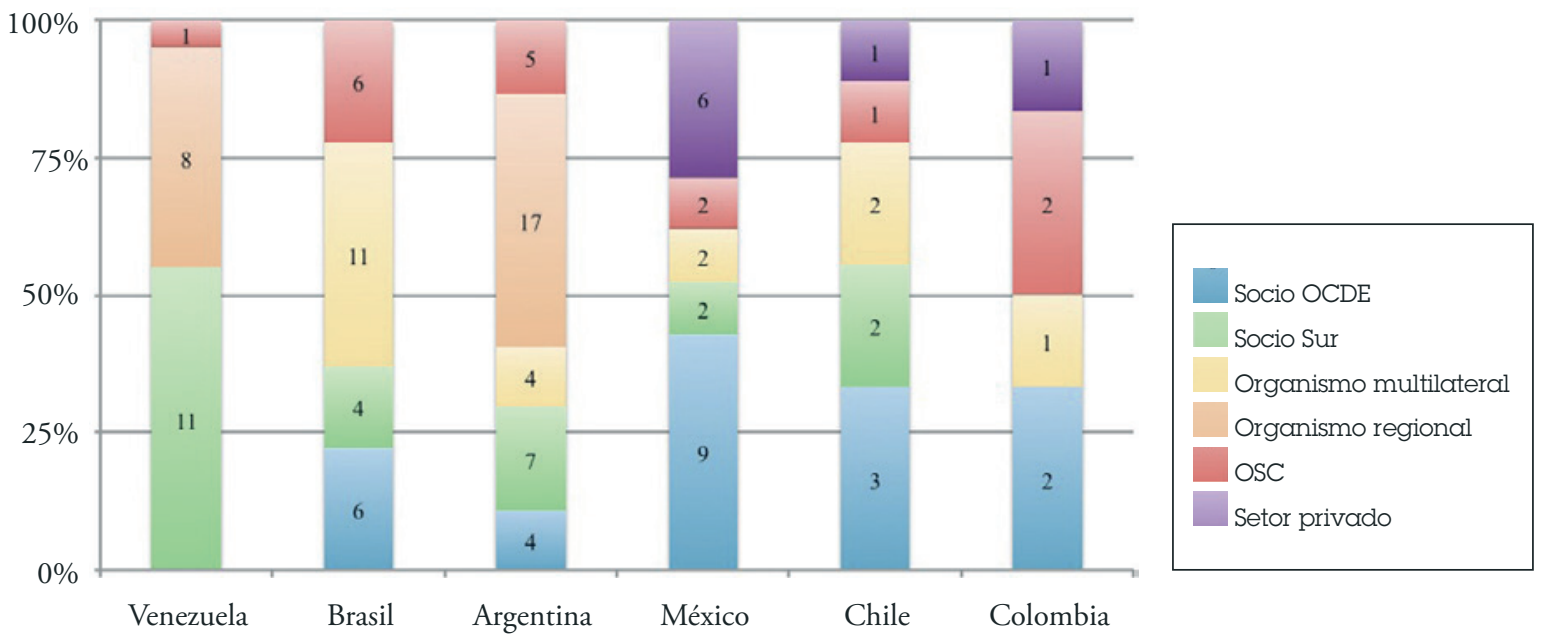

Fuente: Elaboración propia en base a Malacalza (2016)

se destaca los realizados en materia de salud con Venezuela y Cuba (MALACALZA, 2016).

Una segunda matriz, el idealismo defensivo, tuvo como eje una fuerte retórica anti-imperialista, anti-estadounidense y crítica de la MINUSTAH. En el caso de Venezuela, la CSS aspiró a ejercer influencia geopolítica subregional, constituyéndose en un instrumento del proyecto bolivariano en la cuenca del Caribe. Ello se reflejó en una mayor densidad de las triangulaciones Sur-Sur-Sur (55\% de las acciones) y en las articulaciones de cuño regional-sur-sur ( $40 \%$ de las acciones) en el marco del ALBA-TCP. ${ }^{11}$

Por su parte, la tercerización prestigiosa buscó en la triangulación con el Norte una oportunidad para la obtención de reputación internacional. Los casos de México, Chile (con ciertos matices) y Colombia, que suelen ser caracterizados como "economías abiertas" (el primero y el segundo, miembros de la OCDE, y el tercero, aspirante a miembro), compartieron la

11. La regionalización vía ALBA-TCP se fundamentó principalmente en tres vectores: el suministro de petróleo; la CSS en proyectos de infraestructura, salud, educación y desarrollo social; y la articulación de un esquema de asociación económica post-capitalista basado en los proyectos de Grannacionales del ALBA. El ingreso de Haití al Fondo PetroCaribe en 2007 institucionalizó por la vía regional el suministro petrolero y el fondo destinado a la CSS, al que se incorporó Cuba, brindando asistencia técnica en salud y educación. preferencia por la triangulación Norte-Sur-Sur, además del impulso a las asociaciones público-privadas (PPP). Las articulaciones con socios del Sur no estuvieron ausentes, salvo en acciones realizadas entre sí, como las del fondo Chile-México.

\section{Concepciones de descrrollo y cooperación sur-sur: la selectividad}

La heterogeneidad de la CSS regional fue también un reflejo de las diferentes propuestas y modelos de desarrollo que coexistieron en la región. A diferencia de la década del noventa, cuando las políticas mercado-céntricas ocupaban el centro de la escena, en la década pasada, pareciera haber existido un consenso en la literatura sobre la existencia de una tríada de modelos.

Altmann y Aravena (2008) observan que las decisiones sobre la economía, el modelo productivo, la apertura hacia el comercio externo y los regímenes de inclusión social dieron cuenta de tres líneas diferenciadas en la región: el desarrollo centrado en el mercado, el equilibrio entre Estado y mercado (con especial énfasis en la inclusión social) y el desarrollo centrado en el Estado. 
Almeida (2014), en tanto, identifica un modelo "bolivariano", más intervencionista, estatista y rupturista respecto de la economía de mercado; un modelo de países "reticentes dubitativos", basados en las capacidades reguladoras de sus Estados, pero que cuentan también con el mercado y el sector empresarial, a la vez que poseen regímenes de inclusión social; y un modelo de países "globalizados" que abogaron por esquemas de libre comercio, una activa iniciativa empresarial en la economía y políticas alineadas al mundo desarrollado.

En un nivel más micro, la adopción de diferentes propuestas de desarrollo implicó, además, una definición respecto a qué políticas seguir en materia productiva, social, institucional, de infraestructura etc. En función de ello, existieron matices relacionados a las peculiaridades de cada estructura económica, pudiéndose observar una más amplia gama de propuestas de acuerdo a la especialización de cada país: desde las neodesarrollistas con énfasis en la internacionalización de empresas nacionales (Brasil), las mercado internistas, las agroexportadoras, las estatistas centradas en la administración de la abundancia petrolera (Venezuela y Ecuador), las centradas en la exportación de recursos minerales (el cobre en Chile), hasta las centradas en el ensamble y la complementación con Estados Unidos (México).

La experiencia acumulada por los PIs en determinados foros internacionales se relacionó también con sus desarrollos en determinadas especializaciones temáticas. Así, en el tema agroalimentario y de biocombustibles, Brasil conservó un reconocimiento en el ámbito multilateral que le valió la designación de funcionarios brasileños a cargo de importantes organismos. ${ }^{12}$ Argentina fue también una voz autorizada en seguridad alimentaria, como

12. Ello se ha plasmado en las elecciones, en 2011, de José Graziano da Silva, ex Ministro de Seguridad Alimentaria de Lula Da Silva, como director general de la FAO y, en 2013, de Roberto Carvalho de Acevedo como director general de la Organización Mundial del Comercio (OMC) miembro del G20, como participante de Conferencias sobre Seguridad Alimentaria de la Organización para la Agricultura y la Alimentación (FAO) y como presidente temporal del G77. México, en tanto, se proyectó como una economía abierta alineada a los estándares normativos promovidos por la OCDE. ${ }^{13}$ Colombia también fue cultor de influencias dentro de esta organización, presidiendo, desde 2009, el Task Team sobre CSS (TTSSC). Además, a partir de su experiencia en el control de fondos ilícitos, obtuvo un reconocimiento en la Organização das Naçóes Unidas (ONU), presidiendo el Comité de Lucha contra la Corrupción. Chile mantuvo también cierta influencia en la discusión de la agenda de desarrollo, siendo Santiago sede de la histórica Comissão Econômica para a América Latina e o Caribe (CEPAL). Además, buscó incidir en la agenda con nuevos temas como lucha contra el cambio climático ${ }^{14}$ e igualdad de género. ${ }^{15}$ Por su parte, Venezuela conservó su influencia en recursos energéticos como miembro de la Organización de Países Exportadores de Petróleo (OPEP).

Como demuestra el gráfico anterior, la selectividad en el caso de Haití da cuenta de un clara correlación entre las diferentes especializaciones en políticas públicas y los perfiles sectoriales de la CSS de los PIs en estudio. Siguiendo a Almeida (2014), pueden trazarse tres perfiles. En el esquema "bolivariano", como expresión de la diplomacia petrolera, la CSS de Venezuela estuvo orientada en el 67\% de sus acciones a obras de infraestructura (construcción de carreteras, aeropuertos, espacios públicos, edificios públicos, etc.) y a proyectos de energía (totaliza nueve, la mayor cantidad de un país de la

13. Desde 2006, su Secretario General es el mexicano José Ángel Gurría Treviño, ex Secretario de Hacienda de Ernesto Zedillo.

14. El ex presidente Ricardo Lagos fue designado enviado especial de la ONU en esta materia.

15. Chile tuvo un papel protagónico en la fundación de ONU Mujeres en 2010, organismo especializado en la lucha a favor de la igual de género. La primera dirección de ese organismo fue ocupada por la actual presidenta chilena Michelle Bachelet. 
Gráfico 2 -Porcentaje de acciones de CSS con Haití según sectores (2005-2015)

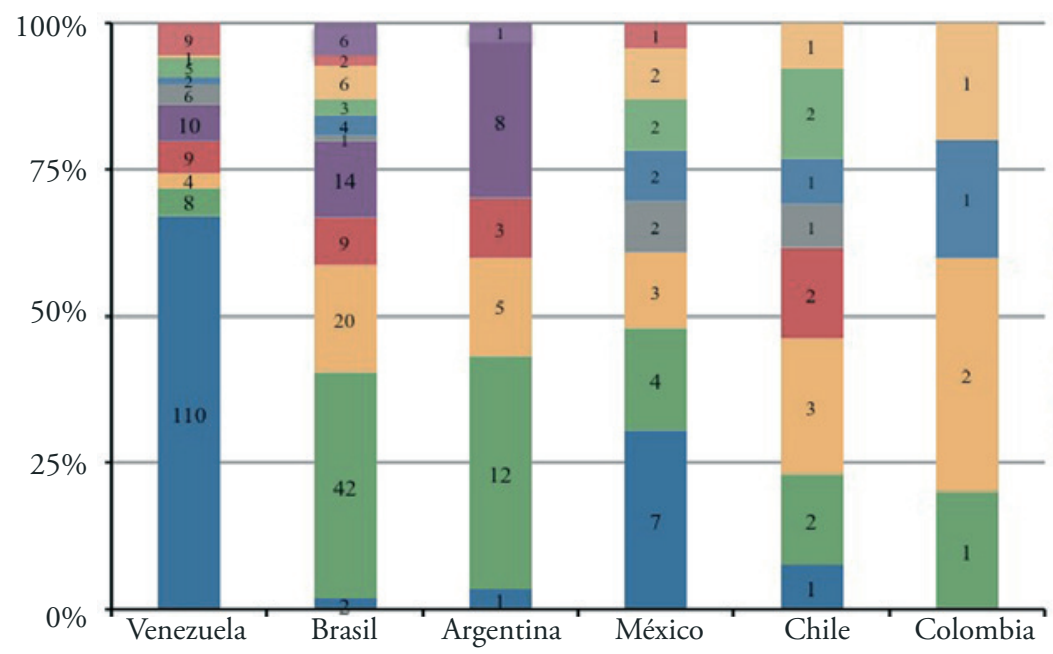

Fuente: Elaboración propia en base a Malacalza (2016)

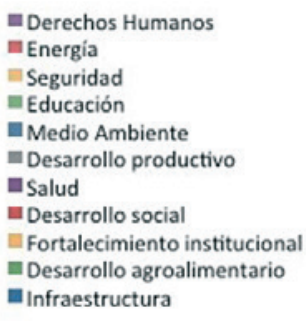

región) (MALACALZA, 2016).

Entre los países "reticentes dubitativos", la CSS de Brasil se correspondió con el ascenso de dos diplomacias, la agrícola y la de salud. De hecho, un $39 \%$ de sus proyectos fueron orientados al desarrollo agrícola y alimentario y un $27 \%$ a la salud en Haití. Se destacaron, allí, dos instituciones modelo de políticas publicas como Empresa Brasileira de Pesquisa Agropecuária (EMBRAPA), en materia agroalimentaria, y Fundação Oswaldo Cruz (FIOCRUZ), en materia de salud (MILANI; BARAN; BRAS 2016). ${ }^{16}$ En una menor escala, Argentina destinó un $40 \%$ de su cooperación a la seguridad alimentaria, siendo su eje el fortalecimiento de la pequeña agricultura familiar (proyecto ProHuerta).

Los países "globalizados" como México, Chile y Colombia (economías del Pacífico) presentaron como rasgo común el trabajo en el desarrollo del sector privado haitiano, las inversiones y el comercio exterior (ejes de fortalecimiento institucional y desarrollo productivo), para lo cual establecieron alianzas público-privadas. No obstante, también se

16. Entre los proyectos de Brasil, se destaca un proyecto para el fortalecimiento de las producciones de arroz, castańa de caju, mandioca, maíz y café, un programa de huertas en Fond-desNègres y un proyecto de inmunización y salud pública (MILANI et al, 2016). diferenciaron en función de sus experiencias: Chile en educación infantil inicial y agricultura familiar, México en medio ambiente, salud y fronteras, y Colombia en la producción de café (MALACALZA, 2016) ${ }^{17}$ En suma, la selectividad de la CSS de acuerdo a temas y asuntos de política publica tuvo estrecha relación con las ideas de desarrollo que se promovieron y los sectores productivos y de desarrollo social favorecidos por las políticas domésticas. Las ideas de la CSS, a su vez, se correspondieron a los diferentes esquemas de desarrollo: "bolivarianos", "reticentes" y "globalizados".

\section{Conclusiones}

Un PI es un estado que busca algún tipo de aventura en el sistema internacional. No se contenta con ser espectador. Sea a través del pragmatismo autonómico, el idealismo defensivo o la tercerización prestigiosa, los intereses de la CSS que siguen los PIs se corresponden con esa aspiración de la política exterior. Aún cuando no existe la coherencia en estos esquemas, es posible advertir la prevalencia

17. Para más información sobre el Fondo Chile-México véase (AGENCIA DE COOPERACIÓN INTERNACIONAL, 2006) 
de diferentes modos de triangulación y de selectividad de la CSS en función de la mayor o menor densidad de unos vínculos sobre otros y la fuerte conexión de la CSS con los esquemas de política exterior y las concepciones de desarrollo imperantes.

En ese sentido, el análisis comparativo de los casos de Venezuela, Brasil, Argentina, México, Chile y Colombia en Haití permite confirmar las dos hipótesis de esta investigación. Los PIs hacen CSS para salir de la insignificancia, pero ese modus operandi no es uniforme. Si se analiza la variable "triangulación", es posible advertir tres matrices de CSS: la diversificada (pragmatismo autonómico), la contestataria con mayor densidad de vínculos Sur-Sur-Sur (idealismo defensivo) y la adaptativa con mayor densidad de vínculos Norte-Sur-Sur (tercerización prestigiosa). Mientras que, si se observa la "selectividad", se advierte la aspiración de los PIs a ejercer un liderazgo selectivo en determinados temas o "nichos": por ejemplo, los "reticentes dubitativos" en agroalimentos y salud, el "bolivariano" en infraestructura y energía, y los "globalizados" en comercio exterior.

Las motivaciones de los PIs para hacer CSS son cambiantes a lo largo del tiempo y en función de los donantes. Es adecuado afirmar que los cambios no sólo están relacionados al ámbito doméstico y a las concepciones de las elites, sino también a un sistema internacional en constante transformación. Las causas o motivaciones no son estáticas, sino que se encuentran ligadas a la dinámica política de intereses e ideas que circulan externa e internamente y pueden converger o contradecirse. Las políticas de CSS, como efectos de esas dinámicas, no son coherentes y hasta pueden resultar ambiguas. ¿Cuándo y cómo cambian las fuentes y motivaciones de la CSS? ¿Qué efectos producen esos cambios en las políticas exteriores de CSS? El cómo (el proceso) contribuye a explicar el porqué (las motivaciones), que también es necesario para comprender los efectos (los resultados). Se trata, pues, de tres elementos de análisis de la cooperación internacional que resultan útiles y convergentes para una agenda de investigación sobre el desempeño de los PIs en la CSS.

\section{Referências}

AGENCIA DE COOPERACIÓN INTERNACIONAL. Ministerio de Relaciones Exteriores. Fondo conjunto de cooperación Chile - México: integración, amistad y desarrollo. Chile: AGCI, 2006. Disponível em: <http://www.agci.gob.cl/ fondo_chile_mexico/docs_fondo_chi_mex/presentacion_chile_mexico_sept_2012.pdf>._Acesso em: 10 jul. 2016.

ALMEIDA, Paulo Roberto. A grande fragmentação na América Latina: globalizados, reticentes e bolivarianos. Carta Internacional, v. 9, n. 1, p. 79-93, 2014.

ALTMANN, Josette; ARAVENA, Francisco Rojas. Las paradojas de la integración en América Latina y el Caribe. Espańa: Siglo XXI, 2008.

BISCHOFF, Paul-Henri. External and domestic sources of foreign policy ambiguity: South African foreign policy and the projection of pluralist middle power. Politikon, v. 30, n. 1, p. 183-201, 2003

CHASE, Robert S.; HILL, Emily B.; KENNEDY, Paul. Pivotal states and US strategy. Foreign Affairs-New York, v. 75, p. 33-51, 1996.

CLAXTON, Brooke. The place of Canada in post-war organization. Canadian Journal of Economics and Political Science, v. 10, n. 04, p. 409-421, 1944.

COOPER, Andrew F. Niche diplomacy: a conceptual overview. En Niche Diplomacy. Palgrave Macmillan UK, 1997. p. 1-24.

DAHL, R. Análisis político moderno. Barcelona: Fontanella, 1976.

FLEMES, Daniel. O Brasil na iniciativa BRIC: soft balancing numa ordem global em mudança. Revista Brasileira de Política Internacional, v. 53, n. 1, p. 141-156, 2010.

GLAZEBROOK, G. Det. The middle powers in the United Nations system. International Organization, v. 1, n. 02, p. 307-318, 1947

HIRST, Mónica. América Latina y la Cooperación Sur-Sur: reflexiones conceptuales y políticas. In: AYLLÓN, Bruno; SURASKY, Javier. (Coord.). En la cooperación sur-sur en latinoamérica: utopía y realidad. [S.1.]: Catarata, 2010, p. 17-39.

HOLBRAAD, Carsten. The role of middle powers. Cooperation and Conflict, v. 6, n. 1, p. 77-90, 1971.

HOLBRAAD, Carsten. Middle powers in the international system. New York: St. Martin’s Press, 1984.

HUELSZ, Cornelia. Middle power theories and emerging powers in international political economy: a case study of Bra- 
zil. PhD Thesis. Manchester: University of Manchester, 2009.

HURRELL, Andrew. Hegemony, liberalism and global order: what space for would-be great powers? International Affairs, v. 82 , n. 1, p. 1-19, 2006.

JONES, Rodney W.; HILDRETH, Steven. Emerging powers: defense and security in the third world. New York: Praeger, 1986.

KEOHANE, Robert O. After hegemony: cooperation and discord in the world political economy. Princeton: Princeton University Press, 2005.

LIMA, María Regina Soares de. Brasil como país intermedio: imprecisión conceptual y dilemas políticos. In: TOKATLIÁN, Juan (Comp.). India, Brasil y Sudáfrica: el impacto de las nuevas potencias regionales. Buenos Aires: Libros del Zorzal, p. 169-190, 2007.

LUMSDAINE, David Halloran. Moral vision in international politics: the foreign aid regime, 1949-1989. Princeton: Princeton University Press, 1993.

MALACALZA, Bernabé. Unidad y dispersión: balance de una década de Cooperación Sur-Sur Latinoamericana en Haití. Cuadernos Técnicos de Trabajo sobre Cooperación Sur-Sur, Vol. 1., julio. Madrid: Secretaría General Iberoamericana, 2016. Disponivel em: < www.cooperacionsursur.org/images/ guia-2016.pdf>. Accesso em: 30 jul. 2016.

MELISSEN, Jan. The new public diplomacy: between theory and practice. The New Public Diplomacy, Palgrave Macmillan UK, 2005. p. 3-27.

MILANI, Carlos R. S.; BARAN, Katarzyna; BRAS, Hugo. Brazil's development cooperation in Haiti since 2010: actor, interests and outcomes. Final Report. Five years after earthquake in Haiti: lessons from South-South Cooperation (the cases of Brazil, Chile and Mexico). CEPAL-Instituto Mora, 2016.

MORGENTHAU, Hans. A political theory of foreign aid. American Political Science Review, v. 56, n. 2, p. 301-309, 1962.

MUHR, Thomas. Beyond 'BRICS': ten theses on South-South cooperation in the twenty-first century. Third World Quarterly, vol. 37, no 4, p. 630-648, 2016.

NEL, Philip. Redistribution and recognition: what emerging regional powers want. Review of International Studies, v. 36, n. 04, p. 951-974, 2010.

NOLTE, Detlef. Potencias regionales: conceptos y enfoques de análisis: el debate político. Revista Iberoamericana de Análisis Político, v. 4, n. 6/7, p. 17-35, 2007.

NOLTE, Detlef. How to compare regional powers: analytical concepts and research topics. Review of International Studies, v. 36, n. 04, p. 881-901, 2010.

POTTER, Evan H. Niche diplomacy as canadian foreign policy. International Journal, v. 52, n. 1, p. 25-38, 1996.

RYERSON, Christie; DEWITT, David. Middle powers and regional security. In: CONFERENCE EMERGENT
POWERS AND REGIONAL SECURITY: THE EXPERIENCE OF IBSA (INDIA, BRAZIL, SOUTH AFRICA). Buenos Aires: Universidad de San Andrés, 2006

SANTANDER CAMPOS, Guillermo. Identidades e intereses en la cooperación Sur-Sur: los casos de Chile, Venezuela y Brasil. Madrid: Catarata, 2016.

SCHOEMAN, Maxi. South Africa as an emerging middle power. African Security Review, v. 9, n 3, p. 47-58, 2000.

SOGGE, David. Dar y tomar ¿Qué sucede con la ayuda internacional? Barcelona: Icaria, 2002.

SPINOZA, B. Ética. Madrid: Alianza Editorial, 1987.

VEEN, A. Maurits van der. Ideas, interests and foreign aid. Cambridge: Cambridge University Press, 2011.

YIN, Robert K. Applications of case study research: applied social research methods series. London: Sage Publications, 1993. 